\title{
The driver vigilance telemetric control system (DVTCS): Investigating sensitivity to experimentally induced sleep loss and fatigue
}

\author{
Jillian Dorrian, Nicole Lamond, Karolina Kozuchowski, ANd Drew Dawson \\ University of South Australia, Adelaide, South Australia, Australia
}

\begin{abstract}
Vigilance technologies are used in the Australian rail industry to address the risks associated with driver sleepiness and fatigue. The aim of this study was to investigate whether a new device, designed to detect lowered states of arousal using electrodermal activity (EDA), would be sensitive to experimentally induced sleepiness and fatigue. Fifteen individuals ( 7 of them female, 9 male; $18-32$ years of age) spent 3 consecutive days in the laboratory, which included 1 night of sustained wakefulness $(28 \mathrm{~h})$. The participants completed a 10-min psychomotor vigilance task (PVT) and fatigue and sleepiness ratings every $2 \mathrm{~h}$, and a 30-min driving simulator every $4 \mathrm{~h}$. As was expected, simulated driving, PVT, and subjective ratings indicated increasing levels of sleepiness and fatigue during sustained wakefulness. The EDA device output did not coincide with these findings. The results indicated that the EDA indicator was not sensitive to increased sleepiness and fatigue at the levels produced in the present study.
\end{abstract}

Over the last century, a significant body of research has established the potential occupational health and safety risks associated with sleep loss and fatigue (reviewed in Dinges \& Kribbs, 1991; Harrison \& Horne, 2000; Kleitman, 1963). Studies have demonstrated that the magnitude of this impairment is not trivial. After remaining awake for a single night (20-24 consecutive hours), performance impairment reaches levels that are equivalent to those for someone with a blood alcohol concentration (BAC) twice the legal driving limit in Australia (i.e., $2 \times .05 \%=.10 \%$ BAC; Lamond \& Dawson, 1999).

Due to their schedules, train drivers often experience sleep of reduced duration and quality (Foret \& Latin, 1972; Pilcher \& Coplen, 2000; Roach, Reid, \& Dawson, 2003) and elevated fatigue levels (Härmä, Sallinen, Ranta, Mutanen, \& Müller, 2002). Several investigations have recorded incidences of drowsing and uncontrolled sleep attack during driving (Cabon, Coblentz, Mollard, \& Fouillot, 1993; Torsvall \& Åkerstedt, 1987). Clearly, such research raises important questions about driver fatigue and safety. Negative outcomes associated with fatigue include increased fuel use (due to reduced alertness and planning and consequent suboptimal brake use), missed signals, failures to sound the horn at grade crossings, and increased emergency brake applications and speed violations (Dorrian, Hussey, \& Dawson, 2007; Dorrian, Roach, Fletcher, \& Dawson, 2006, 2007; Thomas \& Raslear, 1997). Moreover, rail accident investigations have identified work-related fatigue (Pearce, 1999; Zhou, 1991) and the inability to maintain wakefulness (Kogi \& Ohta, 1975; Lauber \& Kayten, 1988) as contributing factors.
Most trains currently have at least one vigilance system built in to determine whether drivers are alert and fit for work. The most common system requires drivers to push a button to acknowledge a timed alarm. If drivers do not acknowledge the light/tone, the emergency brake system brings the train to a stop. Despite such systems, however, the potential for fatigue-related incidents remains an issue in the Australian rail industry, particularly for single-driver operations in urban and freight services. As a result, there is increasing interest in research on technological devices to help counter fatigue-related crashes.

Improvements to vigilance systems have already been tried out in certain sectors of the Australian rail industry. Specifically, on particularly vulnerable corridors, the signal rate of the vigilance protection system has been manipulated, and/or the system has been changed to a choice reaction time approach. In some single-driver (driveronly) operations, the amount of time between required responses has been reduced (i.e., a smaller interstimulus interval [ISI]). This provides more frequent prompts to driver vigilance. Indeed, research has shown that reductions in ISI can render tasks more sensitive to (and are therefore more likely to detect) the effects of sleep loss (Williams \& Lubin, 1967). It has also been argued that tasks requiring repetitive responses at a fairly high rate are particularly sensitive to the effects of sleep loss (Dinges, 1992; Dinges \& Powell, 1985). In addition, on one system, station protection devices have been added, requiring drivers to acknowledge the system by pressing a second button when the train is approaching a station (i.e., choice reaction time). This serves to further engage the

J. Dorrian, jill.dorrian@unisa.edu.au 
driver by requiring him or her to make a conscious decision before pressing the button. Since research suggests that simple, monotonous tasks may reduce motivation to perform (Horne \& Pettitt, 1985), this increased complexity may improve performance by renewing interest and motivation.

When such improvements to existing vigilance systems are considered, two primary issues should be acknowledged. The first issue is that of habituation versus novelty. It is possible for drivers to habituate to a stimulus-response device over time, so that the response becomes automatic and no longer requires increased attention for device compliance. In this way, the nature of the task changes, becoming less interesting and complex. It may, therefore, no longer produce the same motivation to perform (Horne \& Pettitt, 1985). We have anecdotal evidence to suggest that this may already have occurred with some vigilance systems. This has several implications. New devices may provide initial improvement due to novelty, but efficacy will likely decrease to some extent over time as habituation occurs. Furthermore, increasing signal rate (decreasing ISI) may speed up the habituation process.

The second issue is complexity versus distraction. Increasing the complexity of the vigilance device may reduce habituation. One way this can be done is through making the required response a choice (i.e., adding a second response button, as is the case with the station protection devices described above). This requires a conscious decision and, therefore, a higher degree of driver engagement. However, it is essential that any secondary vigilance system should not distract the driver from the primary task of train operation. Indeed, research has demonstrated that secondary tasks (often referred to as distractor tasks) result in performance impairment on the primary task (Horberry, Anderson, Regan, Triggs, \& Brown, 2006). Moreover, distractability and associated performance deficits are exacerbated by sleepiness (Anderson \& Horne, 2006). Therefore, the issue of possible distraction from the primary task of train driving through the introduction of more complex vigilance systems is an important consideration in the rail industry.

One possible way of improving vigilance technologies without increasing complexity is to make the stimulus contingent on the driver's psychophysical or physiological state. For example, a lowered state of arousal may be used to generate a signal to alert the driver that he/she is fatigued. There have been numerous approaches used in physiological sleepiness monitoring that are now being incorporated into tools for industry. For example, the electroencephalograph (EEG) can be used to record brain waves that are indicative of sleep onset or microsleeps (Åkerstedt \& Gillberg, 1990; Lal \& Craig, 2001; Torsvall \& Åkerstedt, 1987; Wright \& McGown, 2001). Another group of technologies focus on the eyes, measuring eye blinking, closure, movement, and pupil responses. Others measure facial muscle tone, body posture, or head nodding (reviewed in Hartley, Horberry, Mabbott, \& Krueger, 2000; Wright, Stone, Horberry, \& Reed, 2007).

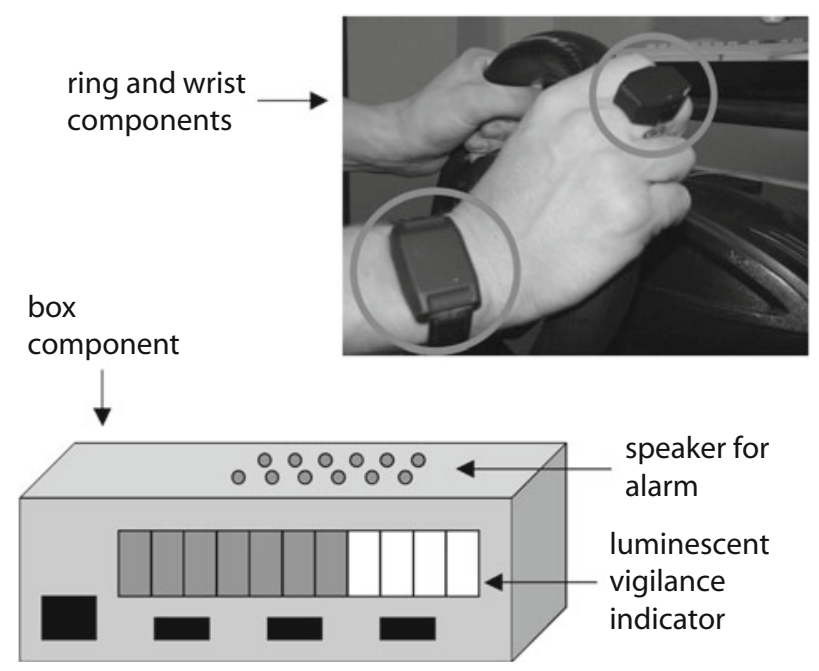

Figure 1. Driver Vigilance Telemetric Control System device. The ring and wrist components were worn by the participant. The box component (remotely receiving signals from the ring and wrist) was located in a separate room and was monitored by an experimenter.

Overall, there are many technologies currently being developed (and some already on the market) that measure various physiological and psychophysiological variables. However, researchers have yet to determine the predictive relationship between these variables and fatigue-related crashes (Hartley et al., 2000). One such technology that has recently been introduced is the Driver Vigilance Telematic Control System (DVTCS; Neurocom, 2002, www .neurocom.ru/en/index.html). This device, which measures the driver's electrodermal activity (EDA), is designed to detect lowered states of arousal and to warn the driver when vigilance levels drop to a critical level (approximately $1 \mathrm{~min}$ before the onset of sleep). The device is worn on the wrist and/or the finger.

The physiological bases for EDA responses are not yet fully understood; however, a variety of research suggests that EDA indirectly measures sweat gland activity (Andreassi, 2000; Dementienko et al., 2000). Eccrine sweat glands, which are most abundant on the palms and soles of the feet, respond weakly to certain levels of heat but strongly to psychological and sensory stimuli. Thus, these areas are useful for EDA measurement (Andreassi, 2000). It has been suggested that EDA "is an accepted vigilance index used in diverse psychophysiological fields" (Miró, CanoLozano, \& Buela-Casal, 2002, p. 105). Indeed, it has been found to be associated with self-reported sleepiness levels, reaction times, core body temperature (Miró et al., 2002), performance errors, and EEG changes that are indicative of drowsiness (Dorokhov, Dementienko, Koreneva, Markov, \& Shakhnarovitch, 1998; Dorokhov et al., 1998c). Studies have also found an association between EDA responses and car crash avoidance (Collet, Petit, Priez, \& Dittmar, 2005). For these reasons, and due to its noninvasiveness and simplicity, EDA has been suggested as a useful measure for sleep deprivation studies (Miró et al., 2002). 
However, a recent field study showed less convincing results. Specifically, Wright and McGown (2001) investigated sleepiness and sleep in aircrews during long-haul flights. Findings indicated that although EDA (skin resistance) changed during the times the pilots were asleep, as compared with when they were awake, the change was gradual. Importantly, the authors suggested that although changes in EDA indicate underlying tiredness, fatigue, and low arousal, this occurs in a general way, rather than being specifically related to sleepiness and sleep. They concluded that skin resistance was too broad a measure to be useful in detecting sleepiness and likelihood of falling asleep.

Despite the scientific debate surrounding the use of EDA measures for sleepiness detection, the DVTCS approach (in particular, EDVTCS, a specific version adapted to the locomotive cab) has received international attention from the rail industry. Approval for the device has been granted by the Ministry of Transport of Russia, and it has been in wide use for 5 years in the Russian railways (Whitlock, 2002). Recent interest in implementing the device has been shown by the rail industry in Australia (Kamensky, 2004) and also in the U.K. (Whitlock, 2002). The manufacturers report that, around the world, approximately 8,000 train drivers use the device (Kamensky, 2004).

The Rail Safety and Standards Board (U.K.) commissioned Quintec to conduct and report on systematic technology information search and to provide a cost/benefit analysis of driver readiness or alertness-monitoring devices (Whitlock, 2002). Of the 13 devices identified, the EDVTCS was considered to be the most developed and the most suitable for possible implementation. The report reviewed published scientific abstracts on EDA provided by the device manufacturers (Dorokhov et al., 1998; Dorokhov et al., 1998a, 1998b, 1998c) and included a feasibility evaluation based on user acceptance, railway context, and scientific criteria. In the report, it was also highlighted that information on the use of the device for rail was not available. In the Rail Safety and Standards Board's response to the Quintec report, it was recommended that the device be tested further in the laboratory or simulator. With an approximate cost per unit of $€ 6,060$ (Whitlock, 2002, Appendix A.10), the decisions around possible device implementation are economically significant.

Therefore, the aim of this laboratory study was to determine whether the DVTCS can be used reliably to assess reduced alertness and sleepiness in fatigued individuals. The device consists of a wrist and a ring component (worn by the individual) that send signals to a box with a luminescent-rule-style vigilance indicator (Figure 1). The indicator counts down as lowered states of arousal are detected. When vigilance, as indicated by ring or wrist EDA, reaches sufficiently low levels, an alarm sounds, and the driver is required to press a response button. This action will reset the device and restart the vigilance countdown. This aspect of the device (the alarm and response button) is similar to the existing vigilance technologies already in place in Australian trains, as described above. Importantly, this study will focus on the EDA portion of the device (i.e., the participants are blind to the luminescent vigilance indicator and are not required to reset the device). This will be outlined further in the Method section. Specifically, the aim of this study was to determine whether the EDA portion of the DVTCS device is sensitive to fluctuations in sleepiness and fatigue related to time of day and increases in time awake.

\section{METHOD}

\section{Study Design}

This was a repeated measures study, in which 15 participants underwent a period of $28 \mathrm{~h}$ of sustained wakefulness (independent variable). Dependent variables, recorded every $2 \mathrm{~h}$, included psychomotor vigilance response times, subjective fatigue, driving simulator performance, and DVTCS output. Within-subjects comparisons were used to investigate the impact of hours awake (experimentally induced fatigue).

\section{Participants}

Fifteen individuals ( 6 of them female, 9 male), 18-32 years of age $(M \pm S D=23.8 \pm 4.0)$, participated in the present study. (Of the 16 originally recruited, 1 was dropped who could not complete the study because of a headache.) The participants were nonsmokers who did not regularly consume large doses of caffeine. Those recruited had no current health problems and were not taking any medication other than an oral contraceptive. All were self-reported

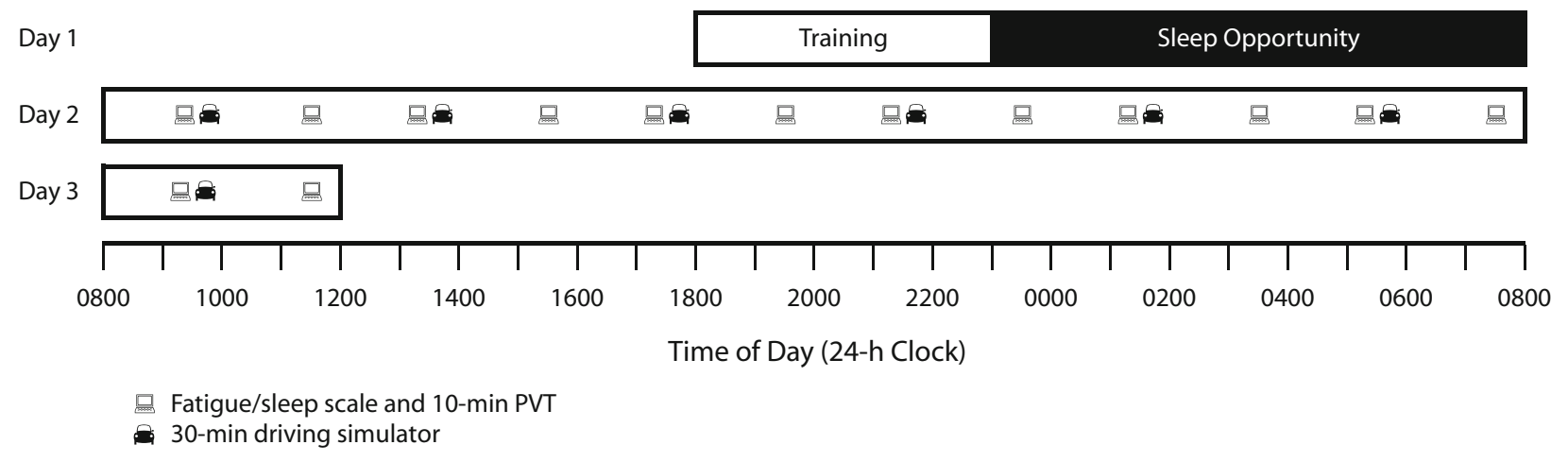

Figure 2. Protocol diagram displaying activities by time of day ( $x$-axis) for each day of the protocol ( $y$-axis). PVT, psychomotor vigilance task. 
good sleepers who did not habitually nap and had not undertaken shift work or transmeridian travel in the past month.

\section{Procedure}

The protocol was approved by the University of South Australia Human Research Ethics Committee. The protocol reflected that used by Lamond and Dawson (1999), mentioned above, in order to ensure that the participants reached levels of fatigue that were measurable and meaningful. That is, after remaining awake for a single night (20-24 consecutive hours), participants in this study demonstrated levels of performance impairment that were equivalent to someone with a BAC twice the legal driving limit in Australia $(0.10 \%$ BAC).

The protocol is displayed in Figure 2. Each participant spent 3 consecutive days in the laboratory. The participants arrived at $1800 \mathrm{~h}$ and were assigned to their individual bedrooms. After they had been provided with verbal and written descriptions of study procedures and rules, the participants completed a short training session to familiarize themselves with the various performance tasks and devices. Following the training session, the participants spent $9 \mathrm{~h}$ in bed $(2300-0800 \mathrm{~h})$ to ensure that they had obtained adequate sleep prior to the experimental period. The participants were awakened at $0800 \mathrm{~h}$ and were allowed to shower and eat breakfast.

Prior to the commencement of testing, a standard montage of electrodes was applied to each participant's face and scalp, to allow continuous monitoring of brain activity (EEG) and eye movements (EOG). The participants remained in their individual rooms, semirecumbent in a chair, and remained awake until $1200 \mathrm{~h}$ the following day, to experimentally induce high levels of sleepiness and fatigue. During this time, the participants completed a 10-min psychomotor vigilance task (PVT) and gave ratings of sleepiness and likelihood of falling asleep every $2 \mathrm{~h}$. Every $4 \mathrm{~h}$, the participants completed a 30 -min bout on a driving simulator.

During each experimental session, room temperature was kept constant at $23^{\circ} \mathrm{C}$. The rooms had no windows, and lighting was dim (25-W lamp, oriented toward the back wall of the room). The participant was required to remain seated at all times and was permitted to move from the chair only to use the driving simulator or to visit the bathroom. Testing was conducted by two researchers. During testing sessions, one researcher, in the room with the participant, administered the questionnaires and ensured that the participant was ready to begin each test. The researcher then went to an adjoining room to monitor the box portion of the DVTCS device (as will be described in more detail below). Meanwhile, a second researcher, positioned in the control room, monitored the participant at all times, using a ceilingmounted camera. This experimenter instructed the participant to start the test and the other researcher to start the DVTCS device recording, via an intercom system. In this way, DVTCS recording was synchronized with the PVT and with the driving simulator.

The participants were not permitted any time in bed or opportunity for sleep. Exercise and the use of baths or showers to enhance alertness were not permitted, and the participants were required to abstain from caffeine and other stimulants for the entire study period. Regular balanced meals (breakfast, lunch, and dinner) and snacks (during the night of wakefulness) were provided every $2 \mathrm{~h}$. Between test sessions, the participants were free to engage in quiet activities, such as reading or watching television.

\section{Psychomotor Vigilance Task}

A 10-min PVT, which measures sustained attention, was used to evaluate behavioral alertness (Dinges \& Powell, 1985; reviewed in Dorrian, Rogers, \& Dinges, 2004). During this test, the participants were required to attend to the LED timer display and to press the response button with the thumb of their dominant hand as quickly as possible after the appearance of the visual stimuli. As per standard methodology, the interstimulus interval (ISI) varied from 2,000 to 10,000 msec.

Since the PVT is reported to have a learning curve of one to three trials (Dorrian et al., 2004), the participants were required to com-
Table 1

ANOVA Results for the Psychomotor Vigilance Task (PVT), Subjective Rating Scales, Driving Simulator, and the Driver Vigilance Telemetric Control System (DVTCS) Device

\begin{tabular}{lccc}
\hline & $d f$ & $F$ & $P$ \\
\hline PVT & 13,182 & & \\
$\quad$ 1/RT & & 34.4 & $<.01$ \\
$\quad$ Lapses & 13,182 & 12.5 & $<.01$ \\
Ratings & & 39.7 & $<.01$ \\
$\quad$ Sleepiness & & 33.0 & $<.01$ \\
$\quad$ Fall asleep & 6,84 & & \\
Driving simulator & & 3.6 & $<.05$ \\
$\quad$ Speed variability & & 3.6 & $<.01$ \\
$\quad$ Off-road & & 7.6 & $<.01$ \\
$\quad$ Incorrect lane & & 4.2 & $<.05$ \\
$\quad$ Collisions & 13,182 & & \\
DVTCS during PVT & & 1.8 & n.s. \\
$\quad$ Average vigilance & & 2.5 & $<.05$ \\
$\quad$ Vigilance zero & 6,84 & & \\
DVTCS during driving simulator & & 0.9 & n.s. \\
$\quad$ Average vigilance & & & \\
$\quad$ Vigilance zero & & & \\
\hline
\end{tabular}

plete three trials during a training session on the night prior to the experimental period. During testing sessions, the participants were seated alone in a room in front of a blank wall. Dependent measures, averaged across each 10-min PVT session, included mean speed (reciprocal of average response latency) and number of lapses (response times exceeding $500 \mathrm{msec}$ ).

\section{Subjective Fatigue}

At the beginning of each test session, subjective fatigue was assessed using the Samn-Perelli Fatigue Checklist (Samn \& Perelli, 1982), a forced-choice scale, ranging from 1 (fully alert, wide awake) to 9 (completely exhausted, unable to function effectively). Prior to the commencement of each driving simulator session, the participants were also asked to rate the likelihood that they would fall asleep on a single-item scale, ranging from 1 (not at all likely) to 7 (extremely likely).

\section{York Driving Simulator}

The York driving simulation program (DriveSim 3.00, York Computer Technologies, Kingston, ON) consists of a PC-based interactive driving simulator. It presents a forward view from the driver's seat of a highway road scene, with standard markings and signs appropriate to the road environment. Participants "drive" using a standard steering wheel and pedals for braking and acceleration. The simulator allows for a number of different scenarios that include changes in wind gusts, patterns of other car interactions, and road conditions (e.g., roadwork). There are no turns, but interactions with other cars and changes in speed postings allow assessment of cognitive and motor impairment. Although the York driving simulator shows minimal practice effects, even with drivers of little driving experience (Arnedt, Wilde, Munt, \& Maclean, 2000), prior to the experiment, the participants performed one practice drive to familiarize themselves with the equipment and scenario. In the present study, the driving simulator was programmed to run for $30 \mathrm{~min}$. The dependent variables chosen for analysis included speed variability, lane drift, collision status (with other vehicles and objects), and off-road driving.

\section{Driver Vigilance Telemetric Control System (DVTCS)}

The device was designed to warn of lowered states of arousal, usually associated with sleepiness or extreme relaxation. During each testing session, the participants wore the DVTCS device on the wrist and finger (Figure 1). Two apparatuses were worn to ensure a constant signal, since both were subject to occasional signal dropout (caused by changes in electrode/skin contact, interference, etc.). As was described 

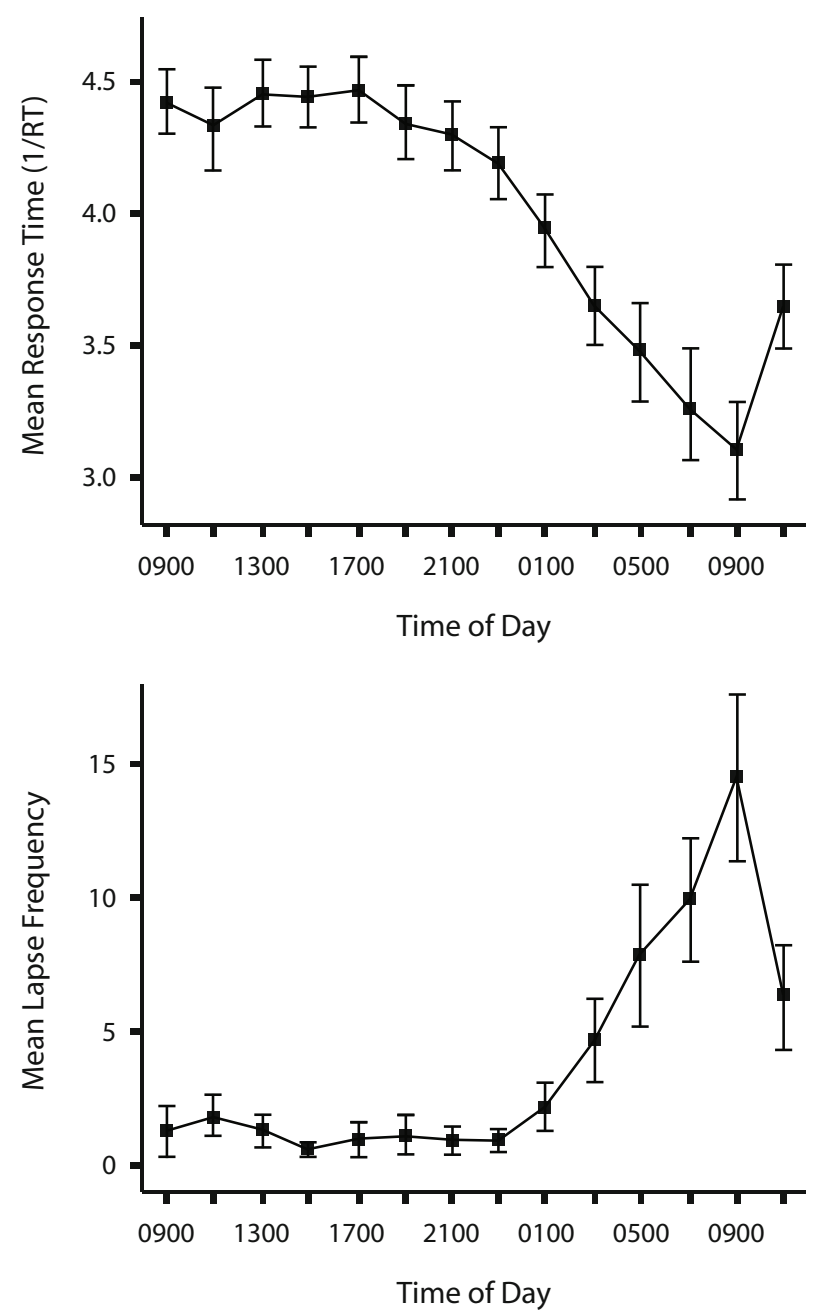

Figure 3. Mean $( \pm S D)$ response time (1/RT, top panel) and number of lapses (RTs $>500$ msec) during the 10-min psychomotor vigilance task (bottom panel).

earlier, the wrist device and ring send signals to a luminescent-rulestyle vigilance indicator, which counts down from a maximum of 15 as lowered states of arousal are detected. When vigilance, as indicated by ring or wrist EDA, reaches sufficiently low levels, an alarm sounds, and the driver is required to press a response button. This action will reset the device and restart the vigilance countdown.

In this study, the alarm was suppressed by a researcher in an adjoining room, who pressed the button to maintain operation of the device. In this way, the experimental participant was blind to his or her vigilance levels as indicated by the device. This important aspect of the protocol allowed us to investigate the signal generated by the EDA portion of the device and to determine whether it fluctuated over the period of sustained wakefulness. This represented a first step toward testing the underlying basis for the device.

The vigilance indicator box was connected to a computer, which recorded the date and time, vigilance levels, and buttonpresses every second. Data collected from this device included the number of times the button was pressed during each PVT session, the mean vigilance scores, and the mean amount of zero vigilance scores during each driving session.

\section{Statistical Analysis}

Systematic changes in each parameter across the experimental session were evaluated using a repeated measures ANOVA with one within-subjects factor (hours of wakefulness) (GLM procedure, SPSS 13 for Mac OSX). The Greenhouse-Geisser procedure was applied to correct for sphericity. Uncorrected degrees of freedom will be reported. Planned comparisons investigated significant differences between Testing Session 1 (0900 h, Day 1) and subsequent testing sessions.

\section{RESULTS}

\section{Psychomotor Vigilance Task}

PVT performance significantly varied across the period of imposed wakefulness. Specifically, response times became significantly longer $(p<.01)$, and the number of lapses during each test significantly increased $(p<.01)$ (see Table 1 and Figure 3). Planned comparisons indicated that relative to Testing Session 1 (0900 h, Day 1), response times were significantly longer $(p<.01)$ during tests that occurred between $2300 \mathrm{~h}$ (Day 1) and $1100 \mathrm{~h}$ (Day 2) and
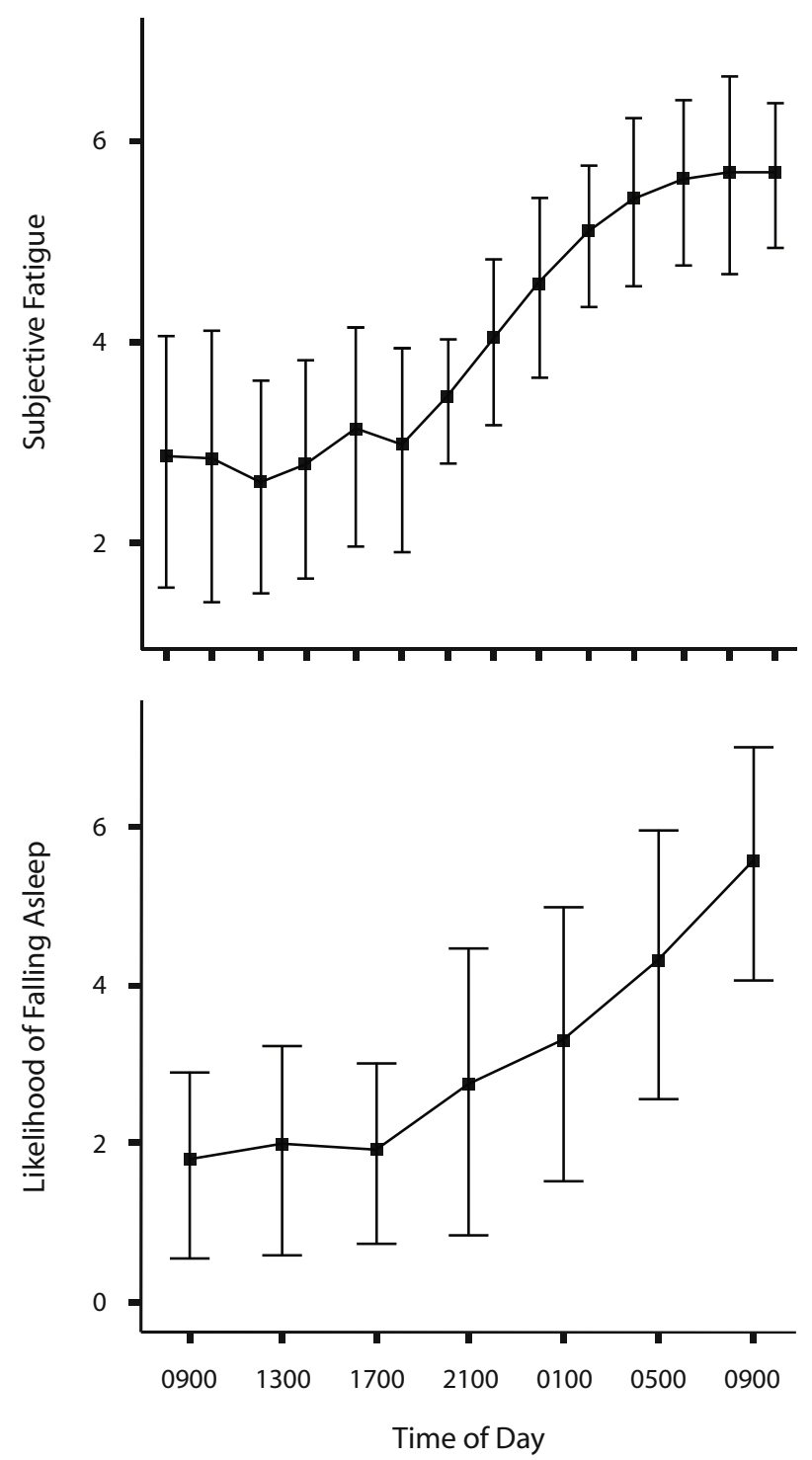

Figure 4. Mean $( \pm S D)$ Samn-Perelli ratings (top panel) and subjective feelings of likelihood of falling asleep (bottom panel). 

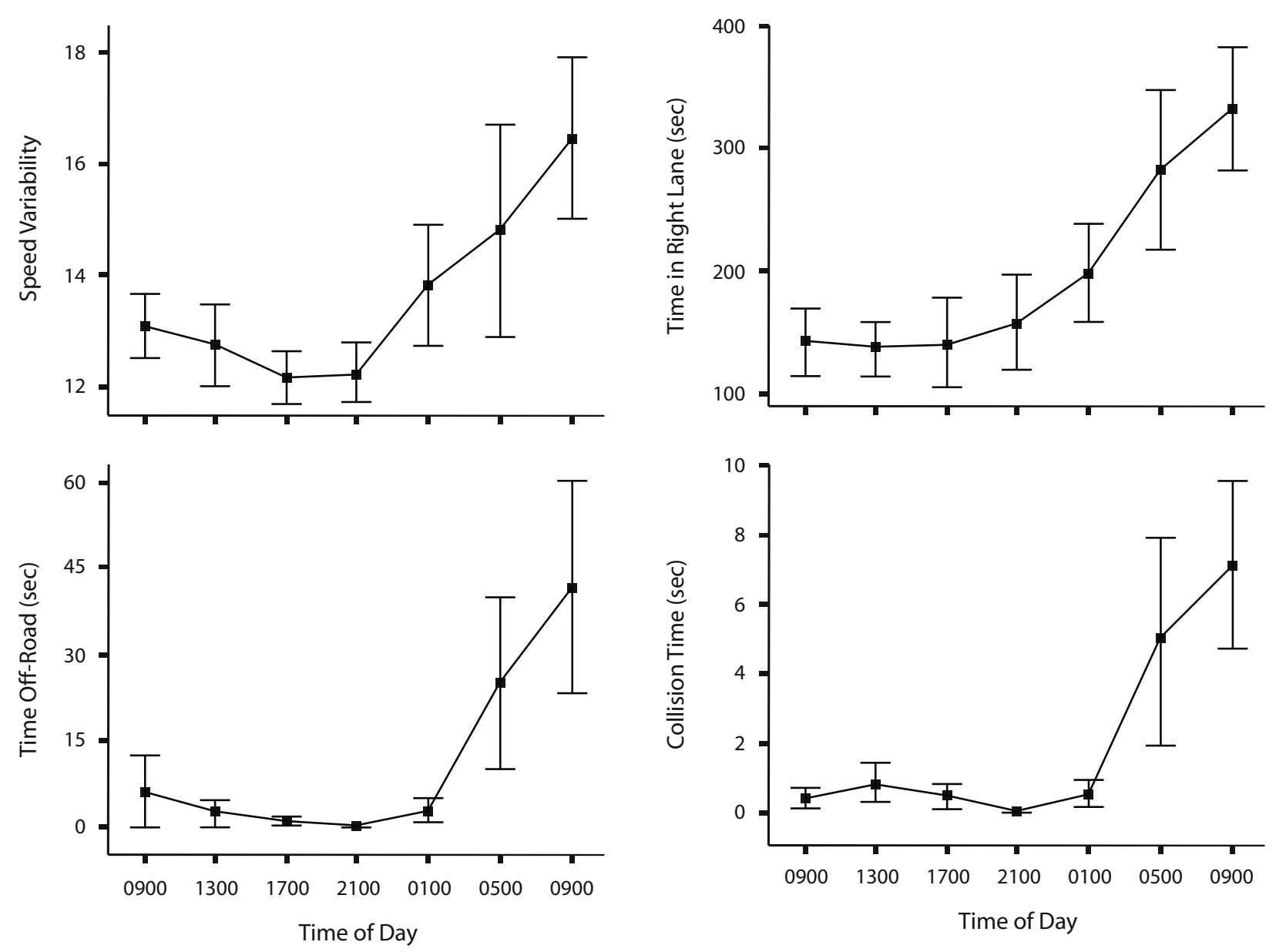

Figure 5. Mean $( \pm S D)$ variability in speed (top left), time spent in the incorrect lane (top right), time spent off the road (bottom left), and time spent colliding with other objects/vehicles (bottom left) during the 30-min driving simulator session.

that lapse frequency was significantly higher $(p<.01)$ during tests that occurred between $0300 \mathrm{~h}$ and $1100 \mathrm{~h}$ (Day 2).

\section{Subjective Ratings}

Subjective ratings indicated that the participants were becoming significantly $(p<.01)$ sleepier throughout the protocol, with the $1300 \mathrm{~h}$ session and sessions after $2100 \mathrm{~h}$ significantly $(p<.05)$ higher than the first test session. Similarly, they felt significantly $(p<.01)$ more likely to fall asleep after $2100 \mathrm{~h}$ (Table 1 and Figure 4).

\section{Driving Simulator}

Analysis indicated that driving performance significantly deteriorated across the period of imposed wakefulness (see Table 1 and Figure 5). Specifically, driving speed became significantly more variable $(p<.05)$, and there was a significant increase in the amount of time that the participants spent (1) driving off-road $(p<.01)$, (2) driving in the incorrect lane $(p<.01)$, and (3) colliding with another car or object $(p<.05)$. Planned comparisons indicated that as compared with the first test session, speed was significantly more variable, and there was a significantly higher number of collisions at $0900 \mathrm{~h}$ (Day 2; $p<.05$ ).
Time spent in the incorrect lane significantly increased from $0100 \mathrm{~h}$ (Day $2 ; p<.05$ ). Time spent driving off-road approached significance at $0900 \mathrm{~h}$ (Day $2 ; p=.066$ ).

\section{DVTCS}

As was described in the Method section, the DVTCS output was recorded every $2 \mathrm{~h}$ during the 10 -min PVT and every $4 \mathrm{~h}$ during the driving simulator task. Data from a small number of test sessions were missing or incomplete. In addition, the DVTCS devices were subject to occasional signal dropout. To control for this, the participants wore a ring and a wrist device. However, on some occasions, both signals failed at the same time. In order to ensure that the results would not be skewed by tasks with missing data or excessive dropout, these tasks were excluded, as will be outlined below.

DVTCS during PVT. Out of a total of 210 tests, 5 tests $(2.4 \%)$ were missing due to problems during data collection. Eight tests $(3.8 \%)$ were missing more than 1 min of data (out of 10) and were therefore removed. Four tests $(1.9 \%)$ were associated with DVTCS signal dropout on both the wrist and the ring for more than a third of the test $(>200 \mathrm{sec})$ and were also removed. This resulted in a total of $8.1 \%$ missing DVTCS data collected during the PVT. 

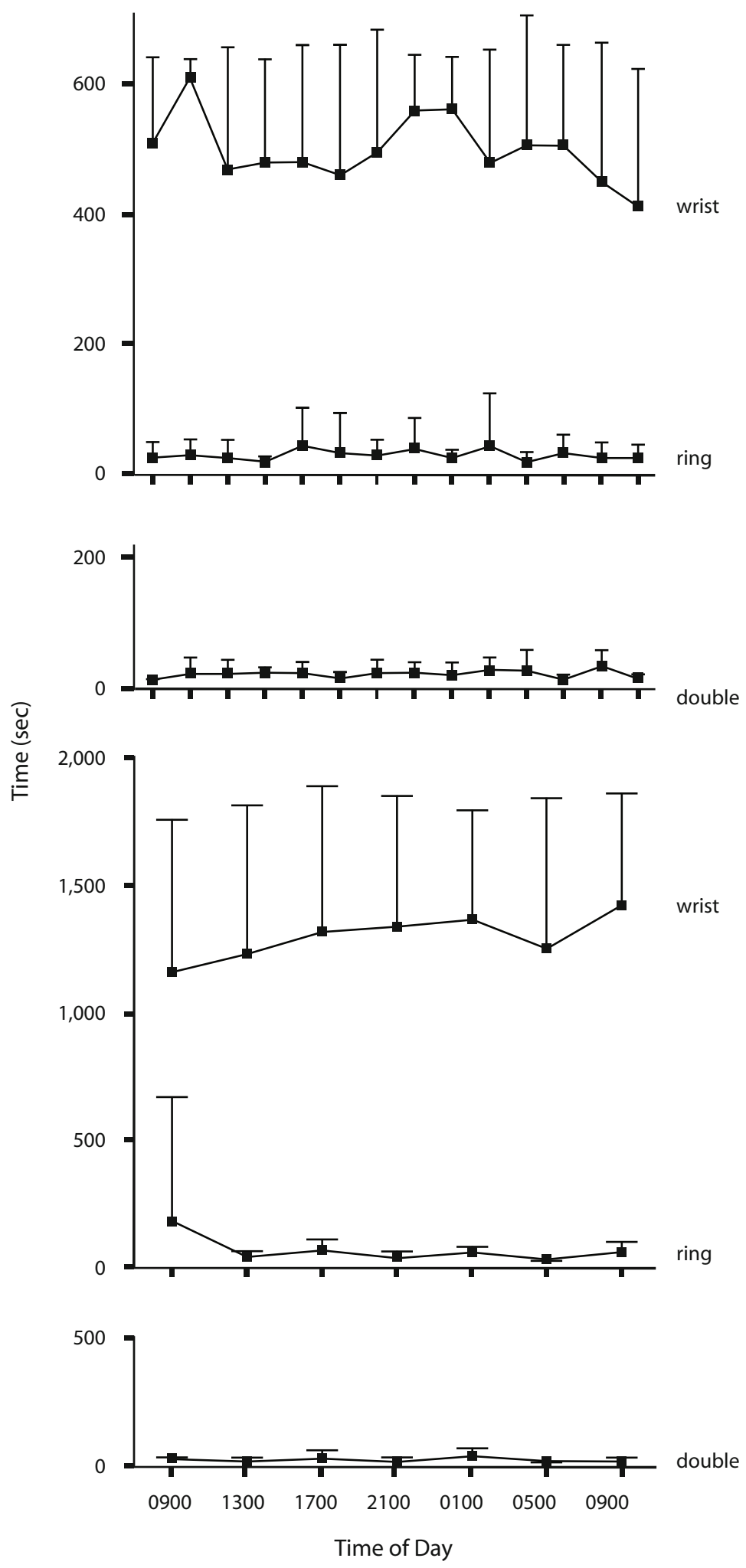

Figure 6. Mean $( \pm S D)$ amount of Driver Vigilance Telemetric Control System data dropout observed during the 10-min psychomotor vigilance task (top panel) and during the driving simulator task (lower panel). 
As can be seen from Figure 6, there was substantially more dropout associated with the wrist than with the ring, and (after removing the outlying 4 tests) double dropout was minimal and was evenly distributed across test sessions. Average vigilance, as indicated by the DVTCS device, did not significantly change across the testing period. The number of seconds that it indicated zero vigilance varied significantly $(p<.05)$, with 2100 and $2300 \mathrm{~h}$ significantly $(p<$ .05 ) higher than $0900 \mathrm{~h}$ on Day 1 (Table 1). However, in real terms, this change represented an average of between 4 and $5 \mathrm{sec}$ out of a test total of $600 \mathrm{sec}$ (Figure 7).

DVTCS during driving simulator. Out of a total of 105 tests, 1 test $(<1 \%)$ was missing due to a problem during data collection. One test $(<1 \%)$ was associated with
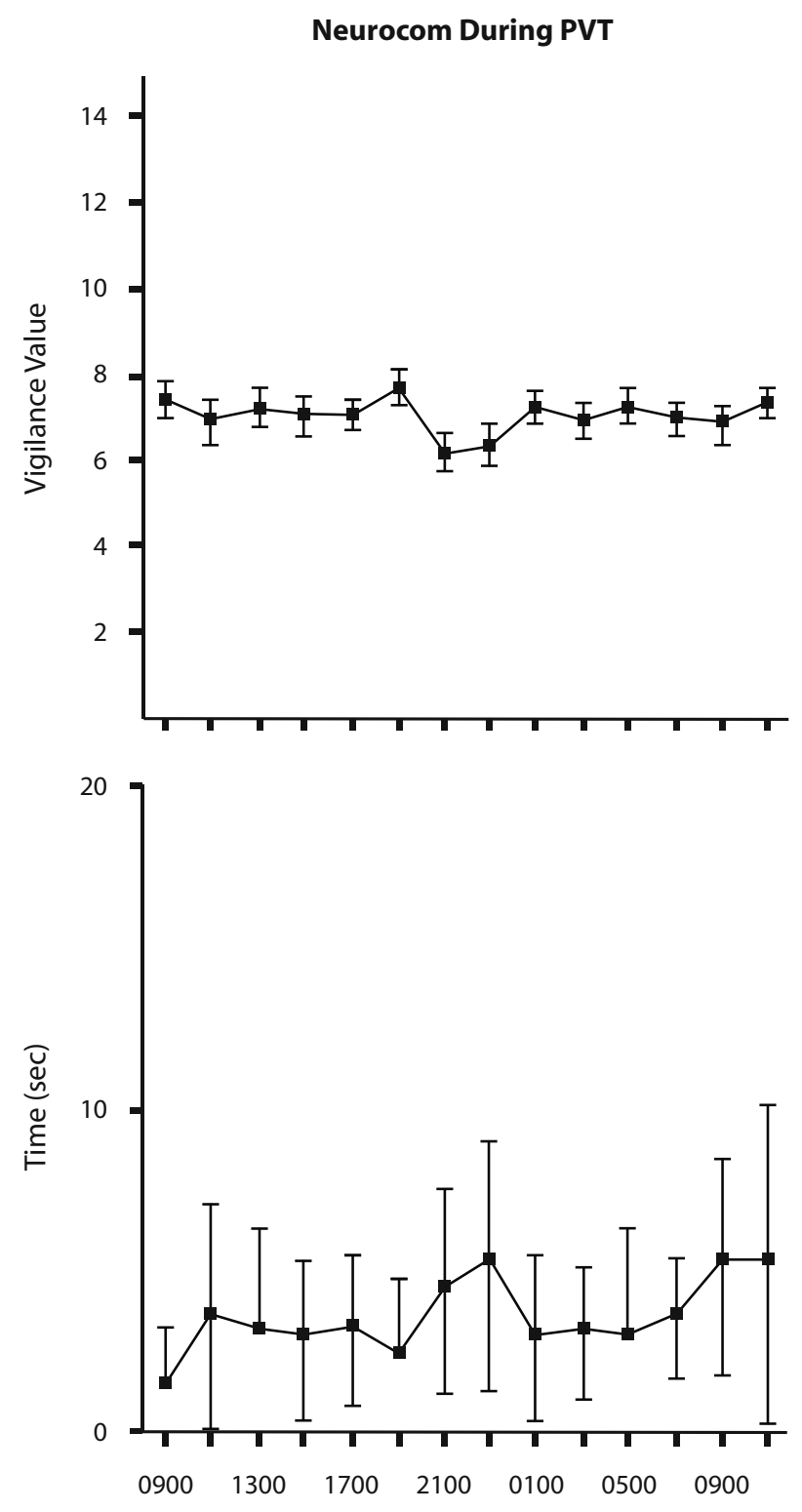

DVTCS signal dropout on both the wrist and the ring for more than $8 \mathrm{~min}$, and was, therefore, removed. This resulted in a total of less than $2 \%$ missing DVTCS data collected during the driving simulator. As can be seen from Figure 6, there was substantially more dropout associated with the wrist than with the ring. Double dropout was minimal and was evenly distributed across test sessions. DVTCS indicators did not significantly $(p<.05)$ vary during the driving simulator task (Table 1, Figure 7).

\section{DISCUSSION}

The specific aims of the study were to assess the validity and reliability of using the EDA portion of the DVTCS
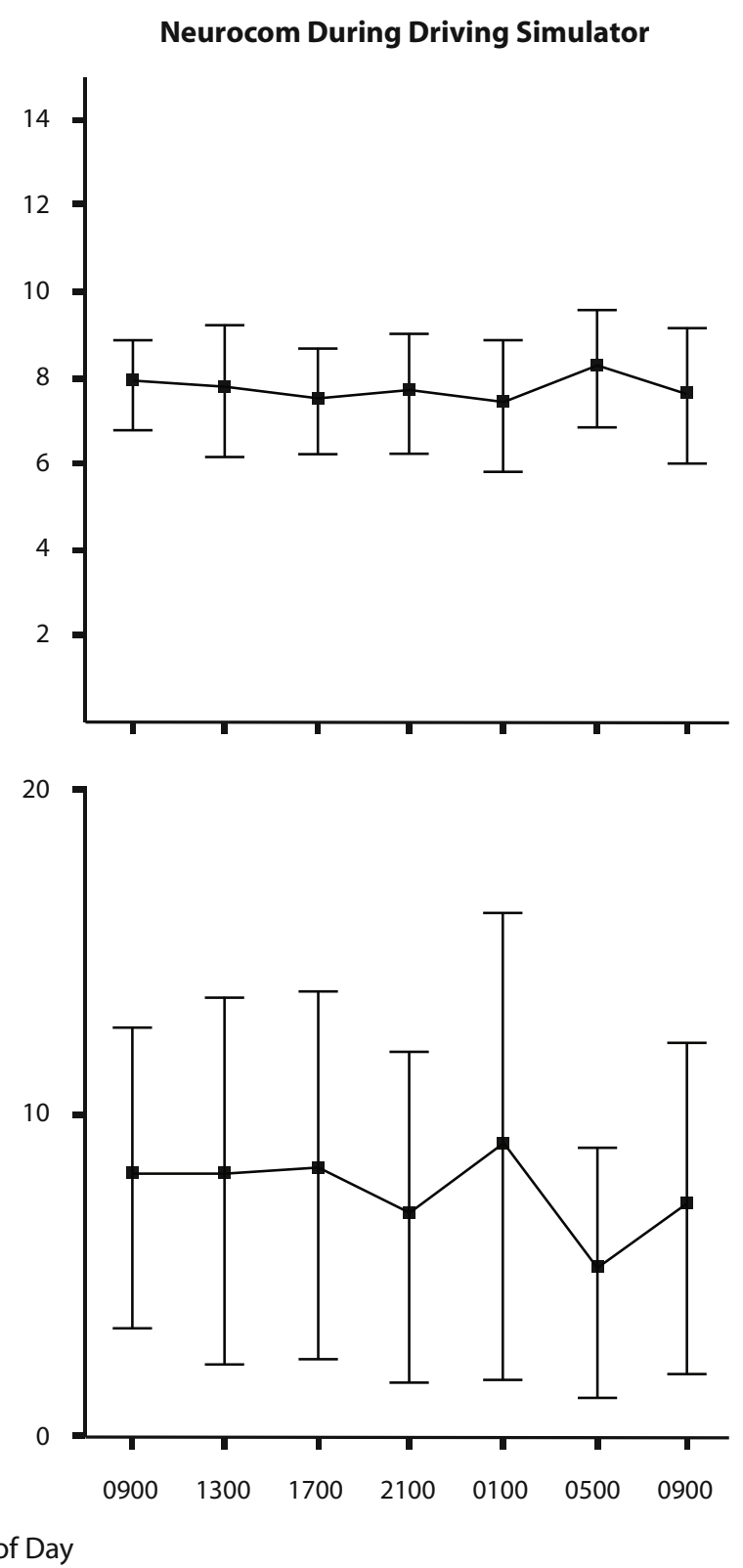

Figure 7. Mean $( \pm S D)$ Driver Vigilance Telemetric Control System vigilance (top panel) and number of seconds that vigilance reached zero (bottom panel) during the psychomotor vigilance task (PVT) (left) and the driving simulator task (right). 
device to assess elevated levels of sleepiness and reduced alertness (i.e., the participants were blind to the luminescent vigilance indicator and were not required to reset the device). This was done by comparing data from the DVTCS device with those from other laboratory tools for assessing sleepiness and fatigue (i.e., subjective ratings, PVT) and a simulated driving task. The study represents an initial step in the DVTCS device validation, exploring the scientific basis for the device (i.e., the EDA portion) under controlled laboratory conditions (i.e., semirecumbent and socially isolated, with constant temperature and lighting).

From a scientific perspective, DVTCS indicators of vigilance indicated very minimal change during the experimental period, despite the fact that PVT, driving simulator, and subjective indicators all reflected measurable impairment. The experimental protocol was specifically chosen to produce measurable and meaningful levels of impairment in participants (as based on Lamond \& Dawson, 1999). Furthermore, this protocol was also run using dim light and no social interaction. Therefore, it could be expected that fatigue would have been exacerbated in the present study. The results suggest that the vigilance indicator part of the DVTCS device is not sensitive to the fatigue levels produced in the present study.

As such, the results appear to be inconsistent with previous (non-peer-reviewed) reports indicating that versions of the device are a "proven, non-invasive, fail-safe vigilance system for train drivers" (Kamensky, 2004, p. 23). There are several possibilities for the inconsistency. First, the algorithm (the calculations by which EDA signals are converted to the 15-point vigilance indicator output and arousal level is determined) may not be optimal. Alternatively, the DVTCS device may be effective; however, positive results may be due to the vigilance portion alone, rather than to the inclusion of the EDA signal used to generate the vigilance stimuli.

Indeed, the vigilance portion of the device consists of a signal generator, which requires drivers to press a button in response to a tone. Failure to respond after a certain time period results in brake application, bringing the train to a stop. The act of responding to the device alerts the driver, encouraging the maintenance of attention. As was previously described, this stimulus-response approach to driver attention is the basis for the vigilance protection systems currently used in Australian trains. Overall, the EDA portion of the device aside, the DVTCS may provide a level of vigilance control superior to those with fixed time intervals, simply due to a more complex vigilance system with a shorter and more variable (unpredictable) warning cycle and inherent novelty value (as outlined in the introduction). Importantly, however, if this is indeed the basis for the reported efficacy of the device, the new aspect of the DVTCS technology (the EDA portion) may not add greatly to existing technology in Australian rail.

Furthermore, the new aspect may not be logistically appropriate. From an operational perspective, the wrist device was associated with a high degree of signal dropout. Signals from the ring device were more consistent, so that using the two signal sources in combination produced a relatively consistent signal. Nevertheless, it is conceivable that outside the controlled laboratory environment, factors such as heat, dirt, and movement may cause increased signal dropout. This is an area for further testing.

The study was an initial step in the DVTCS device validation, exploring the scientific basis for the device (i.e., the EDA portion), using a well-established laboratory protocol for producing measurable and meaningful levels of sleepiness and fatigue. As was expected, simulated driving, the PVT, and subjective ratings indicated increasing levels of sleepiness and fatigue during the experimental period. The EDA device output did not coincide with these findings. The results indicated that the EDA indicator may not be sensitive to increased sleepiness and fatigue at the levels produced in the present study. Future research could examine this further, perhaps directly testing the EDA algorithm in the device and/or testing it under different sleep loss conditions (e.g., more severe ones). The other portion of the device (the vigilance portion) also warrants investigation, in combination with, and possibly also in isolation from, the EDA component. Following this, the practicality and consistency of results under operational conditions (in the cab) need to be systematically tested.

\section{AUTHOR NOTE}

The authors gratefully acknowledge Ryan Higgins (Research Assistant, Centre for Sleep Research, University of South Australia), one of the primary researchers responsible for collection of data in the present study. We also gratefully acknowledge Frank J. Hussey for his advice on some of the more technical aspects of train driving. We also thank Tristan and Jean-Marie (3rd year physiology, University of Adelaide) for their assistance with data acquisition. Correspondence concerning this article should be addressed to J. Dorrian, Centre for Sleep Research, University of South Australia, 7th Floor, Playford Building (P7-35), City East Campus, Frome Rd., Adelaide, SA 5000, Australia (e-mail: jill dorrian@unisa.edu.au).

\section{REFERENCES}

ÅKerstedt, T., \& Gillberg, M. (1990). Subjective and objective sleepiness in the active individual. International Journal of Neuroscience, 52, 29-37.

Anderson, C., \& Horne, J. A. (2006). Sleepiness enhances distraction during a monotonous task. Sleep, 29, 573-576.

ANDREASSI, J. L. (2000). Psychophysiology: Human behavior and physiological response. Mahwah, NJ: Erlbaum.

Arnedt, J. T., Wilde, J. S., Munt, P. W., \& Maclean, A. W. (2000). Simulated driving performance following prolonged wakefulness and alcohol consumption: Separate and combined contributions to impairment. Journal of Sleep Research, 9, 233-241.

Cabon, P., Coblentz, A., Mollard, R., \& Fouillot, J. P. (1993). Human vigilance in railway and long-haul flight operation. Ergonomics, 36, 1019-1033.

Collet, C., Petit, C., Priez, A., \& Dittmar, A. (2005). Stroop colorword test, arousal, electrodermal activity and performance in a critical driving situation. Biological Psychology, 69, 195-203.

Dementienko, V. V., Dorokhov, V. B., Koreneva, L. G., Markov, A. G., Tarasov, A. V., \& Shakhnarovitch, V. M. (2000). The hypothesis of the nature of electrodermal reactions. Human Physiology, 26, 124-131.

DingEs, D. F. (1992). Probing the limits of functional capability: The effects of sleep loss on short-duration tasks. In R. J. Broughton \& R. D. Ogilvie (Eds.), Sleep, arousal, and performance (pp. 176-188). Boston: Birkhäuser.

Dinges, D. F., \& KribBS, N. B. (1991). Performing while sleepy: Effects 
of experimentally-induced sleepiness. In T. H. Monk (Ed.), Sleep, sleepiness, and performance (pp. 97-128). New York: Wiley.

Dinges, D. F., \& Powell, J. W. (1985). Microcomputer analyses of performance on a portable, simple visual RT task during sustained operations. Behavior Research Methods, Instruments, \& Computers, $17,652-655$.

Dorokhov, V. B., Dementienko, V. V., Koreneva, L. G., Markov, A. G., \& ShaKhnarovitch, V. M. (1998). Estimation of the time delay between performance error and its subjective perception during drowsiness. International Journal of Psychophysiology, 30, 95.

Dorokhov, V. B., Dementienko, V. V., Koreneva, L. G., Markov, A. G., Tarasov, A. V., \& ShaKhnarovitch, V. M. (1998a). Hypothesis about the nature of electrodermal reactions. International Journal of Psychophysiology, 30, 267.

Dorokhov, V. B., Dementienko, V. V., Koreneva, L. G., Markov, A. G., Tarasov, A. V., \& Shakhnarovitch, V. M. (1998b). On the possibility of using EDR for estimation of vigilance changes. International Journal of Psychophysiology, 30, 267.

Dorokhov, V. B., Dementienko, V. V., Koreneva, L. G., Markov, A. G., Tarasov, A. V., \& Shakhnarovitch, V. M. (1998c). The peculiarities of electrodermal reactions accompanying the changes of alertness in humans. International Journal of Psychophysiology, 30, 267.

Dorrian, J., Hussey, F., \& DaWson, D. (2007). Train driving efficiency and safety: Examining the cost of fatigue. Journal of Sleep Research, 16, 1-11.

Dorrian, J., Roach, G., Fletcher, A., \& Dawson, D. (2006). The effects of fatigue on train handling during speed restrictions. Transportation Research, 9, 243-257.

Dorrian, J., Roach, G., Fletcher, A., \& Dawson, D. (2007). Simulated train driving: Fatigue, self-awareness and cognitive disengagement. Applied Ergonomics, 38, 155-166.

Dorrian, J., Rogers, N. L., \& Dinges, D. F. (2004). Behavioural alertness as assessed by psychomotor vigilance performance. In C. A. Kushida (Ed.), Sleep deprivation: Clinical issues, pharmacology, and sleep loss effects (pp. 39-70). New York: Dekker.

Foret, J., \& Latin, G. (1972). The sleep of train drivers: An example of the effects of irregular work hours on sleep. In W. P. Colquhoun (Ed.), Aspects of human efficiency (pp. 273-282). London: English Universities Press.

Härmä, M., Sallinen, M., Ranta, R., Mutanen, P., \& Müller, K. (2002). The effect of an irregular shift system on sleepiness at work in train drivers and railway traffic controllers. Journal of Sleep Research, 11, 141-151.

Harrison, Y., \& Horne, J. A. (2000). The impact of sleep deprivation on decision making: A review. Journal of Experimental Psychology: Applied, 6, 236-249.

Hartley, L. R., Horberry, T., Mabbott, N., \& Krueger, G. P. (2000). Review of fatigue detection and prediction technologies. Western Australia: Institute for Research in Safety and Transport. Available at www.ntc.gov.au/filemedia/Reports/ReviewFatigueDetectionand Predict.pdf.

Horberry, T., Anderson, J., Regan, M. A., Triggs, T. J., \& Brown, J. (2006). Driver distraction: The effects of concurrent in-vehicle tasks, road environment complexity and age on driving performance. Accident Analysis \& Prevention, 38, 185-191.

Horne, J. A., \& Pettitt, A. N. (1985). High incentive effects on vigilance performance during 72 hours of total sleep deprivation. Acta Psychologica, 58, 123-139.
Kamensky, G. (2004). A guardian angel called DVTCS-L. Australian Railway Digest, 42, 22-23.

Kleitman, N. (1963). Deprivation of sleep. In N. Kleitman (Ed.), Sleep and wakefulness (pp. 215-229). Chicago: University of Chicago Press.

Kogi, K., \& Онта, T. (1975). Incidence of near accidental drowsing in locomotive driving during a period of rotation. Journal of Human Ergology, 4, 65-76.

LaL, S. K. L., \& Craig, A. (2001). A critical review of the psychophysiology of driver fatigue. Biological Psychology, 55, 173-194.

LAMOND, N., \& Dawson, D. (1999). Quantifying the performance impairment associated with fatigue. Journal of Sleep Research, $\mathbf{8}$, 255-262.

Lauber, J. K., \& Kayten, P. J. (1988). Sleepiness, circadian dysrhythmia, and fatigue in transportation accidents. Sleep, 11, 503-512.

Miró, E., Cano-Lozano, M. C., \& Buela-Casal, G. (2002). Electrodermal activity during total sleep deprivation and its relationship with other activation and performance measures. Journal of Sleep Research, 11, 105-112.

Neurocom (2002). Accessed February 4, 2008, from www.neurocom .ru/en/index.html.

Pearce, K. (1999). Australian railway disasters. Davidson, Australia: IPL Books.

Pilcher, J. J., \& Coplen, M. K. (2000). Work/rest cycles in railroad operations: Effects of shorter than 24-h shift work schedules and oncall schedules on sleep. Ergonomics, 43, 573-588.

RoAch, G. D., ReID, K. J., \& Dawson, D. (2003). The amount of sleep obtained by locomotive engineers: Effects of break duration and time of break onset. Occupational \& Environmental Medicine, 60, e17.

Samn, S. W., \& Perelli, L. P. (1982). Estimating aircrew fatigue: A technique with application to airlift operations (Tech. Rep. SAMTR-82-21). Brooks AFB: USAF School of Aerospace Medicine.

Thomas, G. R., \& RASLEAR, T. G. (1997). The effects of work schedule on train handling performance and sleep of locomotive engineers: A simulator study. Washington, DC: U.S. Department of Transportation and the Federal Railroad Administration.

Torsvall, L., \& ÅKerstedt, T. (1987). Sleepiness on the job: Continuously measured EEG changes in train drivers. Electroencephalography \& Clinical Neurophysiology, 66, 502-511.

Whitlock, A. (2002). Driver vigilance devices: Systems review. Surrey, U.K.: Quintec.

Williams, H. L., \& Lubin, A. (1967). Speeded addition and sleep loss. Journal of Experimental Psychology, 73, 313-317.

Wright, N. [A.], \& McGown, A. (2001). Vigilance on the civil flight deck: Incidence of sleepiness and sleep during long-haul flights and associated changes in physiological parameters. Ergonomics, 44, $82-106$.

Wright, N. A., Stone, B. M., Horberry, T. J., \& Reed, N. (2007). A review of in-vehicle sleepiness detection devices (Published Project Report 157). Berkshire, U.K. Transport Research Laboratory. Available at www.trl.co.uk/store/downloadreport.asp?id $=6171$.

ZHou, D. S. (1991). Epidemiological features and causes of railway traffic accidents. Zhonghua Yu Fang Yi Xue Za Zhi, 25, 26-29.

(Manuscript received November 2, 2007; revision accepted for publication March 25, 2008.) 